\title{
A Magnus Wind Turbine Power Model Based on Direct Solutions Using the Blade Element Momentum Theory and Symbolic Regression
}

\author{
Gustavo Richmond-Navarro, Williams R. Calderón-Muñoz, Richard LeBoeuf, and Pablo Castillo
}

\begin{abstract}
A model of the power coefficient of a mid-scale Magnus wind turbine using numerical solutions of the Blade Element Momentum Theory and symbolic regression is presented. A direct method is proposed for solving the nonlinear system of equations which govern the phenomena under study. The influence of the tipspeed ratio and the number, aspect ratio, and the angular speed of the cylinders on the turbine performance is obtained. Results show that the maximum power coefficient is on the order of 0.2 , which is obtained with two low aspect ratio cylinders, a dimensionless cylinder speed ratio of 2 , and a turbine tip-speed ratio between 2 and 3 . The predicted power coefficient at low tip-speed ratio suggests that a Magnus turbine may be adequate in the urban environment.
\end{abstract}

Index Terms-Blade element momentum theory, Magnus wind turbine, symbolic regression.

\section{NOMENCLATURE}

a Axial flow induction factor

$a^{\prime} \quad$ Tangential flow induction factor

$B \quad$ Number of cylinders

$C_{\text {Pow }}$ Power coefficient

$C_{T} \quad$ Thrust coefficient

$C_{D} \quad$ Drag coefficient

$C_{L} \quad$ Lift coefficient

$C_{m} \quad$ Cylinder moment coefficient

$D \quad$ Cylinder diameter (m)

$F \quad$ Tip-loss model correction factor

$F_{D} \quad$ Drag force $(\mathrm{N})$

$F_{L} \quad$ Lift force $(\mathrm{N})$

$L \quad$ Cylinder length (m)

$L_{0} \quad$ Hub-fixed cylinder length (m)

$P \quad$ Wind turbine power (W)

$P_{\text {Net }} \quad$ Net power output (W)

Manuscript received June 1, 2016; accepted August 21, 2016. Date of publication August 29, 2016; date of current version December 14, 2016. This work was supported by the Instituto Tecnológico de Costa Rica and Ministerio de Ciencia, Tecnología y Telecomunicaciones of Costa Rica. Paper no. TSTE-00411-2016.

G. Richmond-Navarro is with the Escuela de Ingeniería Electromecánica, Instituto Tecnológico de Costa Rica, Cartago 30101, Costa Rica (e-mail: grichmond@ @ec.ac.cr).

W. R. Calderón-Muñoz is with the Department of Mechanical Engineering, University of Chile, Santiago 8370448, Chile, and also with the Energy Center, University of Chile, Santiago 8370448, Chile (e-mail: wicalder@gmail.com).

R. LeBoeuf is with the Department of Engineering and Computer Science, Tarleton State University, Stephenville, TX 76401 USA (e-mail: leboeufrl@gmail.com).

P. Castillo is with the Department of Mechanical Engineering, University of Chile, Santiago 8370448, Chile (e-mail: pablocastillocapponi@gmail.com).

Color versions of one or more of the figures in this paper are available online at http://ieeexplore.ieee.org.

Digital Object Identifier 10.1109/TSTE.2016.2604082

\author{
$P_{\text {rot }} \quad$ Frictional drag power (W) \\ $Q \quad$ Torque $(\mathrm{N} \cdot \mathrm{m})$ \\ $r \quad$ Radius $(\mathrm{m})$ \\ $r_{c} \quad$ Cylinder radius (m) \\ $R_{1} \quad$ Residual \\ $R_{2} \quad$ Residual \\ Re Reynolds number \\ $T \quad$ Axial force (N) \\ $U_{\text {inf }}$ Free-stream air velocity $(\mathrm{m} / \mathrm{s})$ \\ $U_{n} \quad$ Total relative velocity $(\mathrm{m} / \mathrm{s})$ \\ $\alpha \quad$ Angle of attack (rad) \\ $\gamma \quad$ Cylinder aspect ratio \\ $\lambda \quad$ Tip-speed ratio (TSR) \\ $\mu \quad$ Air dynamic viscosity $(\mathrm{kg} / \mathrm{s} \cdot \mathrm{m})$ \\ $\nu \quad$ Air kinematic viscosity $\left(\mathrm{m}^{2} / \mathrm{s}\right)$ \\ $\rho \quad$ Air density $\left(\mathrm{kg} / \mathrm{m}^{3}\right)$ \\ $\Omega \quad$ Turbine angular speed ( $\mathrm{rad} / \mathrm{s})$ \\ $\omega \quad$ Cylinder angular speed $(\mathrm{rad} / \mathrm{s})$ \\ $\bar{\omega} \quad$ Cylinder speed ratio (CSR)
}

\section{INTRODUCTION}

$\mathbf{W}$ HEN a cylinder rotates in a flow field, a lift force perpendicular to the flow direction is generated. This is due to an asymmetric flow pattern and air pressure difference produced by the upper and lower boundary layers separating at different positions [1], which is the well-known Magnus effect.

The use of the Magnus effect to generate lift in cylindrical shaped wind turbine blades has been proposed as an alternative to airfoils. Rotating nonuniform blades have also been used. For example, Mecaro Co. Ltd., a Japanese manufacturer produced a turbine which has spiral shaped fins coiled around its cylindrical blades [2].

In a recent study of Magnus type wind turbines, Bychkov et al. [3] concluded that the Magnus turbine can be exploited in a wide range of wind velocities, from 2 to $40 \mathrm{~m} / \mathrm{s}$, with a rotational velocity 2 to 3 times lower than conventional blade turbines. Luo et al. [2] proposed an analytical solution for Magnus wind turbine power performance based on the Blade Element Momentum (BEM) Theory, ignoring the drag of the rotating cylinders. However, they concluded that the maximum power coefficient of this type of wind turbine needs further confirmation. Using numerical simulation, Sun et al. [4] examined the aerodynamic characteristics of a Magnus wind turbine with various cylinder shapes and found that Magnus type wind turbines normally have low wind energy utilization efficiency. Sedaghat [5] concluded that the Magnus wind turbine power coefficient 
is not as yet promising for urban environments, which includes highly variable flow direction, low speeds and high shear [6]. To improve this performance, local configurations may be considered as proposed by Bourabaa et al. [7].

In this paper, a model of the turbine performance is obtained using the BEM Theory and symbolic regression. The maximum power coefficient of a mid-scale Magnus wind turbine is obtained. Also, the influence of dimensionless parameters, including tip-speed ratio, cylinder speed ratio, aspect ratio and the number of cylinders on the power coefficient is presented.

\section{Mathematical Modeling AND Symbolic RegRession}

\section{A. Direct Method to Estimate Power for Magnus Wind Turbines}

The Actuator Disc Concept [8], explains the energy extraction process in a horizontal axis wind turbine (HAWT) by means of the axial flow induction factor, $a$, which accounts for the wind velocity variation when the air passes through the rotor disc.

Using the linear momentum theorem, Bernoulli's equation and the BEM theory, in which a blade element sweeps out an annular ring of width $\delta r$ (assuming there is no radial interaction between contiguous annuli), the axial force on each annulus is shown in Eq. (1):

$$
\delta T=C_{T} \rho U_{\infty}^{2} \pi r F \delta r
$$

where $C_{T}$ is the thrust coefficient, given by the modified Glauert Correction [9], $\rho$ is the air density, $U_{\infty}$ is the air velocity far upstream, $r$ is the radius to the annular ring, and $F$ is a correction factor from the Tip-Loss Model [9], which takes into account the influence of vortices shed from the cylinder tips on the induced velocity field.

From the angular momentum theorem, the torque on the ring is equal to the rate of change of angular momentum of the air passing through the annulus, as shown in Eq. (2):

$$
\delta Q=4 \rho U_{\infty} \Omega a^{\prime}(1-a) \pi F r^{3} \delta r
$$

where $\Omega$ is the turbine angular speed and $a^{\prime}$ is the tangential flow induction factor, which measures the transfer of rotational motion to the air due to the opposite torque imposed by the rotor upon the air.

Thrust and torque on $B$ cylindrical elements can also be expressed as a function of lift and drag forces, as shown in Fig. 1 and given in Eqs. (3) and (4):

$$
\begin{aligned}
& \delta T=B \cos (\alpha) \delta F_{L}+B \sin (\alpha) \delta F_{D}, \\
& \delta Q=\left(B \sin (\alpha) \delta F_{L}-B \cos (\alpha) \delta F_{D}\right) r,
\end{aligned}
$$

where $\alpha$ is the angle of attack and $F_{L}$ and $F_{D}$ are the lift and drag forces, respectively.

Combining Eqs. (1), (2), (3) and (4) and using the dimensionless lift and drag coefficients, $C_{L}$ and $C_{D}$, the following

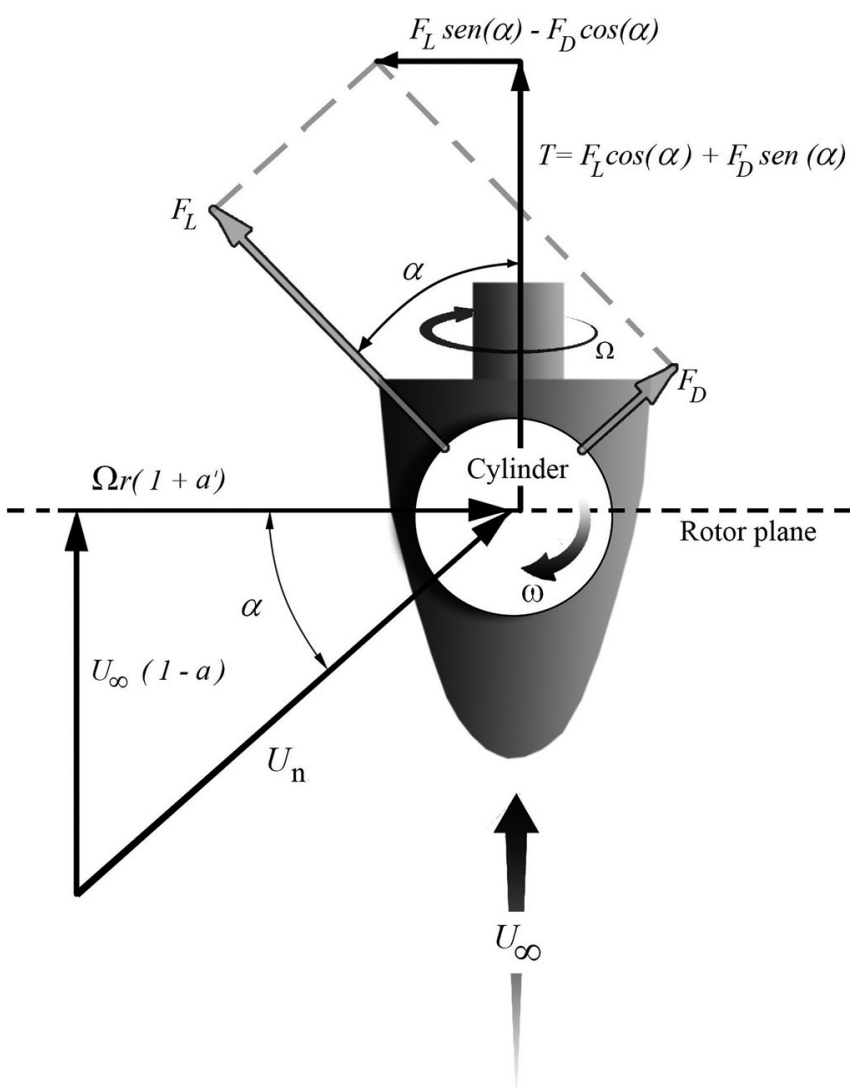

Fig. 1. Local velocities, angles and aerodynamic forces on a Magnus wind turbine cylinder.

nonlinear system of algebraic equations is obtained for $a$ and $a^{\prime}$ :

$$
\begin{aligned}
& 2 C_{T} U_{\infty}^{2} \pi F r-U_{n}^{2}\left(C_{L} \cos (\alpha)+C_{D} \sin (\alpha)\right) B D=R_{1}, \\
& 8 a^{\prime}(1-a) U_{\infty} \Omega r^{2} \pi F-U_{n}^{2}\left(C_{L} \sin (\alpha)\right. \\
& \left.\quad-C_{D} \cos (\alpha)\right) B D=R_{2},
\end{aligned}
$$

where $U_{n}$ is the total relative velocity, $D$ is the cylinder diameter, and $R_{1}$ and $R_{2}$, are residuals which ideally must be zero. The numerical solution of Eqs. (5) and (6) is found by satisfying Eq. (7):

$$
\min _{a, a^{\prime}}\left(\left|R_{1}\right|+\left|R_{2}\right|\right)
$$

In this paper, a Direct Method is proposed for determining flow induction factors that minimize the solution of Eq. 7. It involves evaluation of $\left|R_{1}\right|+\left|R_{2}\right|$ for all the possible values of $a$ and $a^{\prime}$ between 0 and 1 , in increments of $10^{-2}$. Given the interval in which $a$ and $a^{\prime}$ provides the minimum residual, a similar process is repeated to narrow the interval to within $10^{-4}$ and a third pass determines $a$ and $a^{\prime}$ to within $10^{-6}$, which is sufficient to obtain a stable result for the wind turbine power, in all the cases under study. Thus, this process determines the axial and tangential flow induction factors directly. This method provides several benefits relative to an indirect method. It avoids iterative trial and error. It also automatically finds inductions factors within the acceptable range 0 and 1 . Finally, it converges 
for all combinations of input parameters without the need for error criteria.

Given values of the induction factors for each value of $r$, the wind turbine power can be obtained using a discrete set of annular rings of width $\Delta r$, according to the Eq. (8):

$$
P=\sum 4 a^{\prime}(1-a) \rho U_{\infty} \Omega^{2} r^{3} \pi \Delta r,
$$

where the sum includes all $\Delta r$ corresponding to the cylinder length, $L$.

\section{B. Unique Magnus Wind Turbine Characteristics}

In order to describe the Magnus wind turbine properly, energy consumption by the rotating cylinders must be taken into account. An approach is presented by Childs [10], in which the power required to overcome frictional drag for a rotating cylinder is shown in Eq. (9):

$$
P_{\text {rot }}=\frac{1}{2} \pi \rho \omega^{3} r_{c}^{4} L C_{m},
$$

where $r_{c}$ is the cylinder radius and $\omega$ is the angular speed relative to the hub. The moment coefficient for a rotating cylinder, $C_{m}$, is a function of the angular speed and the radius of the cylinder as well as the kinematic viscosity, $\nu$, of the fluid in which the object is immersed.

Using the moment coefficient data provided by Childs for a mid-scale Magnus wind turbine operating in the range of interest, $C_{m}$ may be approximated as shown in Eq. (10):

$$
C_{m}=0.1173\left(\frac{\omega r_{c}^{2}}{\nu}\right)^{-0.219} .
$$

Substituting Eq. (10) into (9) and multiplying by the number of cylinders, $B$, yields the total power consumption, as shown in Eq. (11). Bearing losses of 2.7\%, indicated by Grauers [11], are also included in Eq. (11).

$$
P_{\mathrm{rot}} \approx B \cdot 0.06023 \pi \rho \omega^{3} r_{c}^{4} L\left(\frac{\omega r_{c}^{2}}{\nu}\right)^{-0.219} .
$$

The proposed model adequately captures the exponential behavior of the power consumption as a function of the cylinder angular speed exhibited in the data of Sun et al. [4].

The standard Magnus turbine, in which the entire length of cylinders rotate at a constant rate, can consume more power than that extracted from the wind. This is because, under some operating conditions, the power required to rotate the cylinders is higher than the power captured by the turbine. To eliminate this problem, the inner section of the cylinders can be fixed to the hub and the cylinder angular speed can be selected based on the wind speed. Proper selection of the hub-fixed cylinder section length, $L_{0}$, and the cylinder angular speed, $\omega$, ensures positive power output at all wind speeds, as shown in Fig. 2. The figure compares two Magnus wind turbines with the same geometry ( $L=5 \mathrm{~m}, D=1 \mathrm{~m}, B=3$ ) and angular speed ( $\Omega=30 \mathrm{RPM}$ ). The standard Magnus turbine has a net power consumption at low wind speeds, whereas the turbine with the hub-fixed cylinder section and variable cylinder angular speed has net power output for all spin rates.

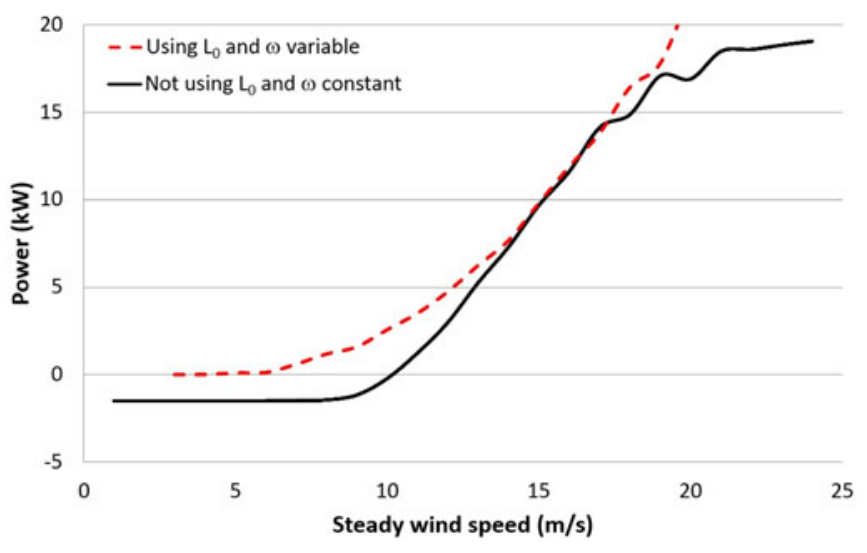

Fig. 2. Two Magnus wind turbines, one standard and other with a hub-fixed length, $L_{0}$, and variable cylinder angular speed, $\omega$.

TABLE I

RANGES OF INPUT PARAMETERS

\begin{tabular}{ccccccc}
\hline \hline B & $\begin{array}{c}U_{\mathrm{min}} \\
(\mathrm{m} / \mathrm{s})\end{array}$ & $\begin{array}{c}\Omega_{\mathrm{min}} \\
(\mathrm{rad} / \mathrm{s})\end{array}$ & $\begin{array}{c}\Omega_{\mathrm{max}} \\
(\mathrm{rad} / \mathrm{s})\end{array}$ & $\begin{array}{c}L_{\mathrm{max}} \\
(\mathrm{m})\end{array}$ & $\begin{array}{c}\omega_{\mathrm{max}} \\
(\mathrm{rad} / \mathrm{s})\end{array}$ & $\begin{array}{c}D_{\max } \\
(\mathrm{m})\end{array}$ \\
\hline 2 & 6 & 3 & 6 & 4 & 600 & 0.2 \\
4 & 7 & 2 & 5 & 5 & 300 & 0.2 \\
6 & 7 & 2 & 5 & 5 & 300 & 0.18 \\
\hline \hline
\end{tabular}

Note: In all cases $U_{\max }=20 \mathrm{~m} / \mathrm{s}, \omega_{\mathrm{m} \text { in }}=200 \mathrm{rad} / \mathrm{s}, D_{\mathrm{m} \text { in }}=$ $0.1 \mathrm{~m}, L_{\mathrm{min}}=2 \mathrm{~m}$ and $L_{0}=0.9 \mathrm{~m}$.

The power curves are obtained simulating the turbine behavior with the proposed Direct Method, using $C_{L}$ and $C_{D}$ curves from Bychkov et al. [3] as input. In the next section, the numerical trials and regression modeling are described.

\section{Symbolic Regression Modeling}

In this section, optimum models are shown based on results of the application of the aforementioned Direct Method to the BEM formulation for several Magnus turbine configurations. Using dimensional analysis, the function for the net power output shown in Eq. (12) can be expressed in terms of dimensionless parameters as shown in Eq. (13).

$$
\begin{aligned}
P_{\mathrm{Net}} & =f\left(U_{\infty}, \Omega, L, \omega, D, \rho, \nu, B\right), \\
\frac{P_{\mathrm{Net}}}{\frac{1}{2} \rho \pi L^{2} U_{\infty}^{3}} & =f\left(\frac{L \Omega}{U_{\infty}}, \frac{\omega D}{U_{\infty}}, \frac{L}{D}, \frac{\rho U_{\infty} L}{\mu}, B\right),
\end{aligned}
$$

where the left hand side is the power coefficient, $C_{\text {Pow }}$, $L \Omega / U_{\infty}=\lambda$ is the tip-speed ratio (TSR), $\omega D / U_{\infty}=2 \bar{\omega}$ is twice the cylinder speed ratio (CSR), $L / D=\gamma$ is the aspect ratio of rotating cylinders, $\rho U_{\infty} L / \mu$ is the Reynolds number and $\mu$ is the dynamic viscosity. Eq. (13) can be written as shown in Eq. (14):

$$
C_{\text {Pow }}=f(\lambda, \bar{\omega}, \gamma, R e, B) .
$$

Symbolic regression was used to obtain the functional $f$.

Using the design of experiments methodology, the set of input parameters shown in Table I was defined. The criterion to define the parameter ranges was to have a positive output power for at 
TABLE II

CORRELATION COEFFICIENTS (CC) AND MEAN ABSOLUTE ERRoRs (MAE) AMONG ALL MODELS

\begin{tabular}{lccc}
\hline \hline & Model for $C_{\text {Pow }}$ & CC & MAE \\
\hline General & $f(\lambda, \bar{\omega}, \gamma, R e, B)$ & 0.95873 & 0.00499 \\
Specific & $f_{2}(\lambda, \bar{\omega}, \gamma, R e)$ & 0.98488 & 0.00341 \\
& $f_{4}(\lambda, \bar{\omega}, \gamma, R e)$ & 0.99261 & 0.00232 \\
Simplified Specific & $f_{6}(\lambda, \bar{\omega}, \gamma, R e)$ & 0.99286 & 0.00237 \\
& $f_{2}(\lambda, \bar{\omega}, \gamma, R e)$ & 0.92979 & 0.00656 \\
& $f_{4}(\lambda, \bar{\omega}, \gamma, R e)$ & 0.93339 & 0.00536 \\
& $f_{6}(\lambda, \bar{\omega}, \gamma, R e)$ & 0.93406 & 0.00720 \\
\hline \hline
\end{tabular}

least $80 \%$ of the combinations and to include values corresponding to an urban environment. Recall that if the design parameters are not selected properly, the output power might not be positive because the power consumption to rotate the cylinders can reduce the net output power to zero. It is necessary to capture this effect in a Magnus turbine model. Moreover, through a process of trial and error it was found that if a higher percentage of positive power combinations are required, the ranges of Table I are drastically reduced such that the ranges exclude values of an urban environment.

A total of 33,500 combinations of the dimensionless parameters of Eq. (14) were generated using the proposed Direct Method. The data was processed with Eureqa predictive analytics software (Nutonian Inc.), which finds functional relationships in data using a machine learning technique called symbolic regression.

\section{RESULTS AND DISCUSSION}

Among the independent variables in Eq. (14), the number of cylinders is the only discrete variable, which affects a stepchange in power output for a given set of the other design variables. As a result, the optimum general function that includes all of the independent variables has a lower correlation coefficient and a higher mean absolute error than those of optimum functions for each cylinder configuration (Table II). Therefore separate functions are found for each of the cylinder configurations analyzed.

\section{A. Cylinder Configuration Specific Models}

For a given number of cylinders, Eq. (14) becomes:

$$
C_{\text {Pow }}=f_{B}(\lambda, \bar{\omega}, \gamma, R e) .
$$

The correlation was improved from about 0.96 for the general model to over 0.98 for the configuration specific models, as shown in Table II.

The Eureqa software provides the relative relevance of each variable, as shown in Fig. 3 . The TSR, $\lambda$, is the principal explanatory variable for the power output of Magnus wind turbines. The same is true of bladed turbines. However, for Magnus turbines, a parameter that is not present in bladed turbines is almost equally important, the CSR, $\bar{\omega}$. This result is in agreement with Bychkov [3], who showed that the Magnus turbine performance is mainly governed by $\bar{\omega}$. The important role of $\bar{\omega}$ is due to the direct effect

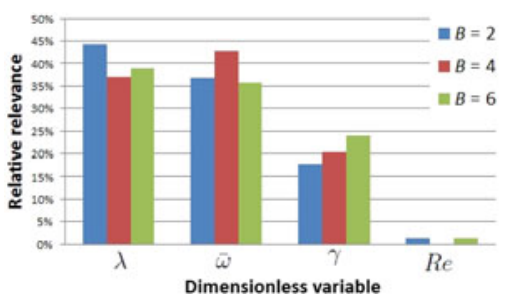

Fig. 3. Relative relevance of each variable across the models.

of cylinder angular speed on the lift and drag coefficients. On the other hand, the high relevance of TSR supports the use of bladed HAWT methods to analyze Magnus wind turbines.

Relative to $\lambda$ and $\bar{\omega}$, the aspect ratio, $\gamma$, is not as significant an explanatory variable of the turbine power output coefficient, $C_{\text {Pow }}$. Besides, the aspect ratio could be constrained due to physical reasons. A Magnus turbine with very low aspect ratio cylinders could be impossible to manufacture because the root of the cylinders could be in contact with adjacent cylinders. A high aspect ratio could lead to significant deflections of the cylinders.

The Reynolds number is not included in the models due to its negligible relative relevance as shown in Fig. 3. The absence of Reynolds number reveals that it was not a fundamental dimensionless term in this study. To understand this, it must be point out that $R e=\rho U_{\infty} L / \mu$, and this research is focused on wind turbines, so the simulations were performed at constant density, $\rho$, and constant viscosity, $\mu$. On the other hand, variations of $U_{\infty}$ and $L$ on mid-scale wind turbines, could be an order of magnitude, which means that $10^{5}<R e<10^{6}$ across all the data. Additionally, for this Reynolds number range, there is not a transition of the flow from laminar to turbulent. Therefore, present results are valid only in the specified $R e$ range.

\section{B. Simplified Models}

Although the specific models have a good correlation with the data, they are complex to interpret. To show the influence of each variable on the system behavior, this section presents simpler models for each cylinder configuration, albeit with a lower correlation coefficient, as shown in Table II. Selecting a minimum correlation coefficient of 0.93 provided models that were sufficiently simple, such that the effects of the design parameters on the power coefficient are clear. Models having higher correlation coefficients were significantly more complicated.

1) Two-Cylinder Configuration $(B=2)$ : The simplified model for the two-cylinder configuration is shown in Eq. (16). In this model there is an inverse relation between $C_{\text {Pow }}$ and the aspect ratio, $\gamma$. Therefore the power coefficient can be plotted as a function of TSR, $\lambda$, and CSR, $\bar{\omega}$, for a fixed $\gamma$, knowing that for higher aspect ratios, all of the $C_{\mathrm{P} \text { ow }}$ values on the surface will be lower. Examples are shown in Fig. 4 for $\gamma=5$ and Fig. 5 for $\gamma=10$, which represents optimistic configurations given the aforementioned aspect ratio constraints.

$$
C_{\text {Pow }}=\frac{1.12 \lambda \bar{\omega}^{2}}{18.2+2.82 \gamma+(3.01+\lambda)^{\bar{\omega}}} .
$$




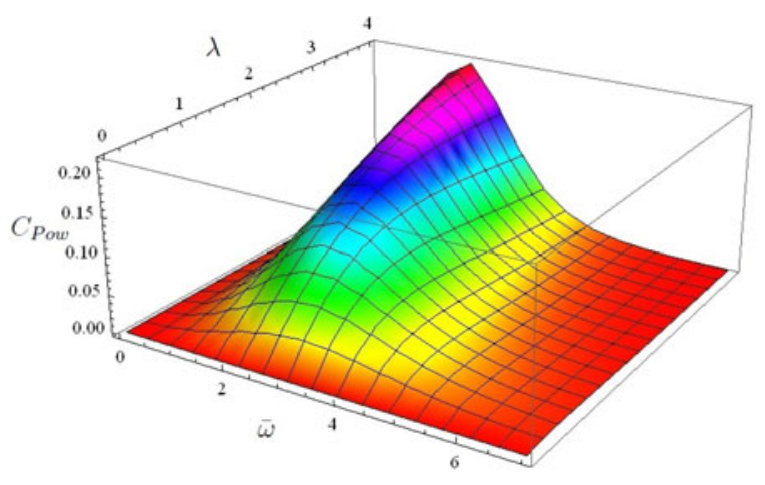

Fig. 4. Power coefficient from simplified model with $B=2$ and $\gamma=5$.

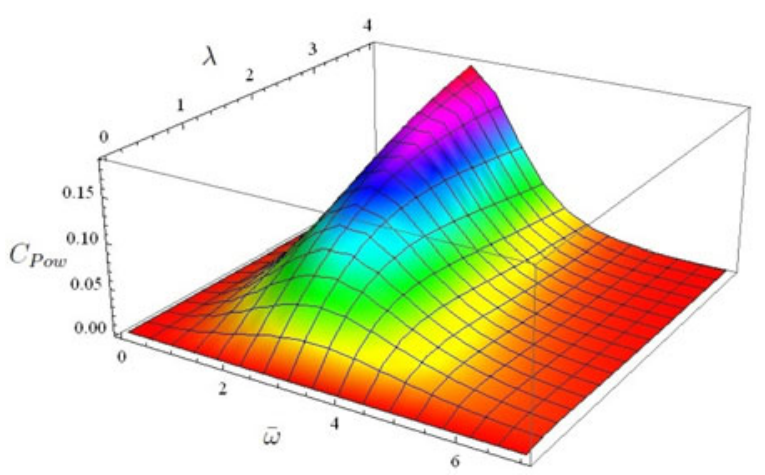

Fig. 5. Power coefficient from simplified model with $B=2$ and $\gamma=10$.

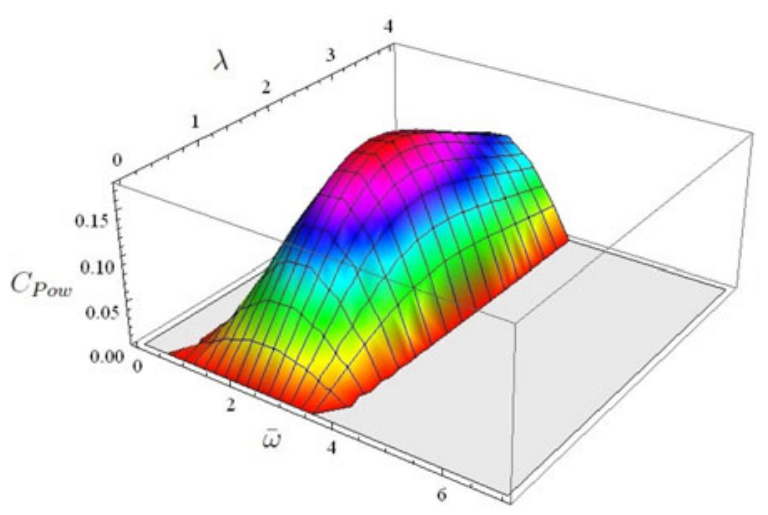

Fig. 6. Power coefficient from simplified model with $B=4$ and $\gamma=5$.

The $\bar{\omega}$ exponent in the denominator ensures that at high cylinder angular speeds, the power becomes zero due to the high energy consumption to rotate the cylinders. Having the maximum at $\bar{\omega}=2$ is in agreement with Sedaghat [5] who specified the range $1.5 \leq \bar{\omega} \leq 2.5$ as the optimum for the spinning cylinder on Magnus type wind turbines. The maximum power coefficient of approximately 0.2 is insensitive to $\lambda$, which is consistent with the aforementioned low relative relevance of the aspect ratio. It is not clear that the peak power coefficient would be found in the TSR range analyzed. However, there is reason to believe that it would not be significantly higher than 4 , since at high $\Omega$ the resultant relative wind velocity of the cylinders would be closer

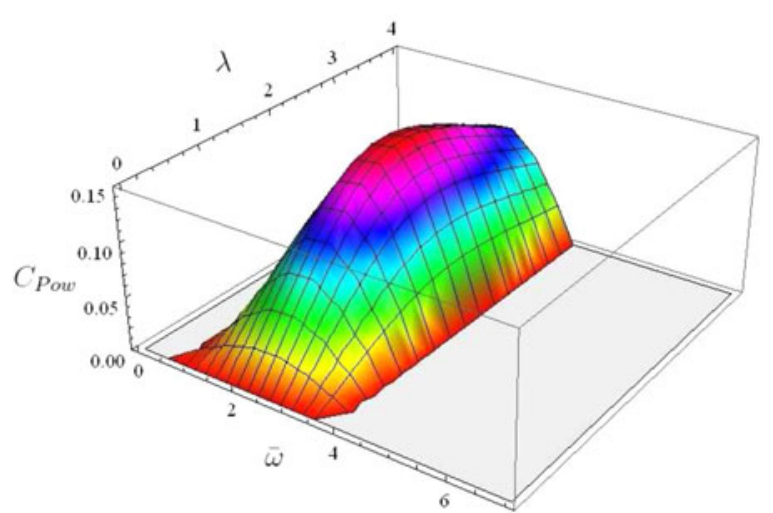

Fig. 7. Power coefficient from simplified model with $B=4$ and $\gamma=10$.

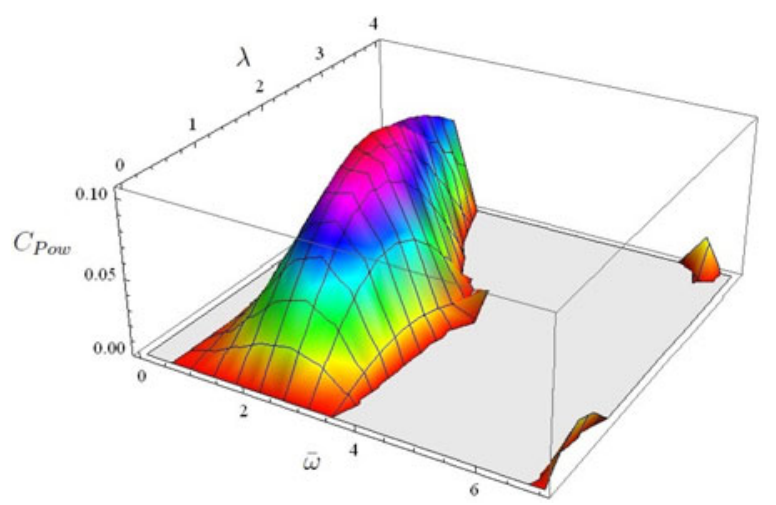

Fig. 8. Power coefficient from simplified model with $B=6$.

to the rotation plane of the turbine and the Magnus force will be in the rotor axial direction, rather than in the circumferential direction.

2) Four-Cylinder Configuration (Case $B=4$ ): The simplified model for the four-cylinder configuration is shown in Eq. (17).

$$
C_{\text {Pow }}=\frac{2.19 \lambda \sin (5.56+\bar{\omega})}{10.8+\gamma+\lambda^{3}} .
$$

Again the model shows an inverse relation between $C_{\text {Pow }}$ and $\gamma$. The power coefficient is shown in Fig. 6 for $\gamma=5$ and Fig. 7 for $\gamma=10$. The surface characteristics are simliar to those of the two-cylinder configuration. Although the function for $C_{\text {Pow }}$ is oscillatory, other peak values are outside the parameter ranges analyzed. The relatively low optimum TSR is in agreement with Bychkov et al. [3]; their study shows that Magnus wind turbines are efficient at low $\Omega$. The maximum $C_{\text {Pow }}$ of approximately 0.15 is lower than that of the two-cylinder configuration. The optimal TSR is between 2 and 3 according to Fig. 6 . The figure clearly shows the reduction of power when the TSR increases beyond the optimum.

3) Six-Cylinder Configuration $(B=6)$ : The simplified model for the six-cylinder configuration is shown in Eq. (18).

$$
C_{\text {Pow }}=0.107 \sin (5.76+\bar{\omega}) \sin (0.392 \lambda \bar{\omega}) .
$$


This simplified model is independent of the aspect ratio, which means that for a high number of cylinders, the threedimensional effects on Magnus force are less significant; the turbine is more like an actuator disc. Since the model is independent of $\gamma$, the surface of Fig. 8 represents, in general, the power coefficient for $B=6$. Again, $\bar{\omega}=2$ and $2 \leq \lambda \leq 3$ coincide with the optimal $C_{\text {Pow }}$ value. This six-cylinder configuration model has a lower $C_{\mathrm{Pow}}$ compared with the other models. The maximum of $C_{\mathrm{Pow}}=0.107$ is evident by inspection of the model equation since the maximum of a sine function is unity.

\section{CONCLUSION}

The influence of number of cylinders, aspect ratio, cylinder speed ratio and turbine tip-speed ratio on the net output power of a mid-scale Magnus wind turbine was investigated to determine a set of parameters that optimize the turbine performance. Several power coefficient models were obtained using numerical solutions and symbolic regression. These models yield the following conclusions:

1) Magnus turbine performance is governed by two main variables, the tip-speed ratio, $\lambda$, and the cylinder speed ratio, $\bar{\omega}$. The significant TSR dependence supports the use of HAWT methods for the analysis of Magnus wind turbines. The CSR dependence is particular to Magnus or Magnus-type turbines.

2) Among the studied cases, the optimum number of cylinders for the Magnus wind turbine is $B=2$. This means that the maximum power coefficient decreases as the number of cylinders is increased, as shown in Figs. 6, 7 and 8, due to the drag increment. The two-cylinder configuration balances energy consumption to generate the cylinder rotation and the energy captured by the turbine.

3) The maximum power coefficient achievable by a midscale Magnus wind turbine is lower than 0.2. This can be achieve at a CSR of $\bar{\omega}=2$ and with a tip-speed ratio, $\lambda$, between 2 and 3 .

The low tip-speed ratio optimum suggests that a Magnus turbine may be adequate in the urban environment. However, more research of cylinder lift and drag is needed to achieve a higher lift without producing an increase in drag, thereby improving the power coefficient of the Magnus turbine and advancing its feasiblility.

\section{REFERENCES}

[1] J. Seifert, "A review of the magnus effect in aeronautics," Progress Aerosp. Sci., vol. 55, pp. 17-45, 2012.

[2] D. Luo, D. Huang, and G. Wu, "Analytical solution on magnus wind turbine power performance based on the blade element momentum theory," J. Renewable Sustainable Energy, vol. 3, 2011, Art. no. 033104.

[3] N. M. Bychkov, A. V. Dovgal, and V. V. Kozlov, "Magnus wind turbines as an alternative to the blade ones," J. Phys.: Conf. Ser., vol. 75, 2007, Art. no. 012004.

[4] X. Sun, Y. Zhuang, Y. Cao, D. Huang, and G. Wu, "A three-dimensional numerical study of the magnus wind turbine with different blade shapes," J. Renewable Sustainable Energy, vol. 4, 2012, Art. no. 063139.

[5] A. Sedaghat, "Magnus type wind turbines: Prospectus and challenges in design and modelling," Renewable Energy, vol. 62, pp. 619-628, 2014.

[6] B. Herrmann-Priesnitz, W. R. Calderón-Muñoz, and R. LeBoeuf, "Effects of urban configuration on the wind energy distribution over a building," $J$. Renewable Sustainable Energy, vol. 7, no. 3, 2015, Art. no. 033106.

[7] N. Bourabaa, E. Delacourt, and J.-L. Menet, "Wind potential evaluation around isolated pitched roof buildings," J. Renewable Sustainable Energy, vol. 7, no. 2, 2015, Art. no. 023111.

[8] T. Burton, N. Jenkins, D. Sharpe, and E. Bossanyi, Wind Energy Handbook, 2nd ed. New York, NY, USA: Wiley, 2011.

[9] P. Moriarty and A. Hansen, "Aerodyn theory manual," Dept. of Energy, Nat. Renewable Energy Lab., Golden, CO, USA, Tech. Rep. NREL/EL500-36881, 2005.

[10] P. R. N. Childs, Rotating Flow. Amsterdam, The Netherlands: Elsevier, 2011.

[11] A. Grauers, "Efficiency of three wind energy generator systems," IEEE Trans. Energy Convers., vol. 11, no. 3, pp. 650-657, Sep. 1996.

Gustavo Richmond-Navarro received the M.Sc. degree in mechanical engineering from Universidad de Chile, Santiago, Chile, in 2014. He is currently an Associate Professor with the Instituto Tecnológico de Costa Rica, Cartago, Costa Rica. His research interests include small-scale wind energy, plasma energy, numerical methods, and CFD simulation.

Williams R. Calderón-Muñoz received the Ph.D. degree in aerospace and mechanical engineering from the University of Notre Dame, Notre Dame, IN, USA, in 2009. He is currently with the Department of Mechanical Engineering, Universidad de Chile, Santiago, Chile. His research interests include energy conversion and storage of renewables, and modeling of physical processes involving heat transfer and fluid mechanics.

Richard LeBoeuf received the B.S. degree in electrical engineering in 1986, the B.S. degree in mechanical engineering in 1987, and the Ph.D. degree in mechanical engineering in 1991 from the University at Buffalo, State University of New York, Buffalo, NY, USA, respectively, and the MBA degree from the University of Maryland, College Park, MD, USA, in 2002. He is currently a Consulting Systems Engineer at a.i. solutions in Maryland.

Pablo Castillo received the M.Sc. degree in aerospace engineering from Aerospace Faculty, Delft University of Technology, Delft, The Netherlands, in 2010. His research interest include new numerical methods to solve stiff integro-differential equations searching for minimum global residual values and modeling of fully coupled algorithm for electrical; mechanical and wireless communication algorithms. 